\title{
Monotonicity of SISO Fuzzy Relational Inference Mechanism with Yager's Class of Fuzzy Implications
}

\author{
Sayantan Mandal and Balasubramaniam Jayaram \\ Department of Mathematics \\ Indian Institute of Technology Hyderabad, \\ Yeddumailaram-502205, India \\ \{ma10p002, jbala\}@iith.ac.in
}

\begin{abstract}
Monotonicity of a Fuzzy Relational Inference (FRI) mechanism has been studied in the literature with implicative form of a rule base where the implication comes from a residuated lattice structure. Here we show monotonicity of an FRI with Yager's class of fuzzy implications which do not form a residuated lattice structure on $[0,1]$.
\end{abstract}

Keywords: monotone rule base, $f$-implications, fuzzy relational inference.

\section{Introduction}

Fuzzy Relational Inferences (FRI) occupy a central position in Approximate Reasoning using fuzzy logic. Two of the well-known Fuzzy Relational Inference mechanisms are the Compositional Rule of Inference (CRI) proposed by Zadeh [10, 4, and the Bandler-Kohout Subproduct (BKS) proposed by Pedrycz [6] based on the earlier work of Bandler and Kohout [2].

Monotonicity is one of the essential properties of an inference mechanism, unavailibility of which leads to an unreliable inference mechanism, see [3], 8]. Monotonicity of an FRI depends essentially on the operations employed in the FRI. The study of monotonicity in an FRI using implicative form of rules has largely been confined to operations that come from a residuated lattice. In this work, we investigate the monotonicity of an FRI where the underlying operations do not come from a residuated structure. Specifically, we study FRIs where the implicative rules are modeled based on the Yager's family of $f$-implications and present conditions underwhich monotonicity of the final output can be ensured.

\section{$2 \quad$ Fuzzy Sets Fuzzy Rules and Fuzzy Logic Connectives}

\subsection{Fuzzy Sets}

Definition 1. If $X$ is a non-empty set then $\mathcal{F}(X)$ is the fuzzy power set of $X$, i.e., $\mathcal{F}(X)=\{A \mid A: X \rightarrow[0,1]\}$.

Definition 2. A fuzzy set $A$ is said to be

P. Maji et al. (Eds.): PReMI 2013, LNCS 8251, pp. 569574 2013.

(C) Springer-Verlag Berlin Heidelberg 2013 
- normal if there exists an $x \in X$ such that $A(x)=1$,

- convex if $X$ is a linear space and for any $\lambda \in[0,1], x, y \in X, A(\lambda x+(1-\lambda) y) \geq$ $\min \{A(x), A(y)\}$.

Definition 3. For an $A \in \mathcal{F}(X)$, the Support, Height, Ceiling and $\alpha$-cut for an $\alpha \in$ $(0,1]$ are, respectively denoted as Supp $A, \operatorname{Hgt} A$, Ceil $A$ and $[A]_{\alpha}$, and are defined as:

$$
\begin{aligned}
\text { Supp } A & =\{x \in X \mid A(x)>0\}, \\
\text { Hgt } A & =\sup \{A(x) \mid x \in X\}, \\
\text { Ceil } A & =\{x \in X \mid A(x)=H g t A\}, \\
{[A]_{\alpha} } & =\{x \in X \mid A(x) \geq \alpha\} .
\end{aligned}
$$

$A$ is said to be bounded if Supp $A$ is a bounded set.

Definition 4. Let $\left\{A_{i}\right\}_{i=1}^{n} \subseteq \mathcal{F}(X)$ be an arbitrary collection of fuzzy sets of $X$. $\left\{A_{i}\right\}_{i=1}^{n}$ is said to form a fuzzy partition on $X$ if

$$
X \subseteq \bigcup_{i=1}^{n} \operatorname{Supp} A_{i} .
$$

In literature, a partition of $X$ as defined above is also called a complete partition.

Definition 5. A fuzzy partition $\left\{A_{i}\right\}_{i=1}^{n} \subseteq \mathcal{F}(X)$ is said to be Ruspini Partition if $\sum_{i=1}^{n} A_{i}(x)=1$ for every $x \in X$.

Definition 6. Let $C \in \mathcal{F}(X)$ and $I \in \mathcal{I}$ be any fuzzy implication.

- We say that a $C^{\prime} \in \mathcal{F}(X)$ is the modification or modified fuzzy set of $C$ by $I$ at a given $\alpha \in[0,1]$ if

$$
C^{\prime}(x)=I(\alpha, C(x)), x \in X .
$$

- Given two convex fuzzy sets $C_{1}, C_{2} \in \mathcal{F}(X)$, we say that $C_{1} \prec C_{2}$ if for any $\alpha \in(0,1]$ it holds that $\inf \left[C_{1}\right]_{\alpha} \leq \inf \left[C_{2}\right]_{\alpha}$ and $\sup \left[C_{1}\right]_{\alpha} \leq \sup \left[C_{2}\right]_{\alpha}$.

The following results show that modification by an $I \in \mathcal{I}$ preserves convexity. We omit the proof of the following results due to space constraints.

Proposition 1. For a convex fuzzy set $C$, a fuzzy implication $I$ and any $\alpha \in[0,1]$, modified fuzzy set $C^{\prime}=I(\alpha, C)$ is also convex.

\subsection{Defuzzification}

Often there is a need to convert a fuzzy set to a crisp value, a process which is called Defuzzification. This process of defuzzification can be seen as a mapping $g: \mathcal{F}(X) \longrightarrow$ $X$. There are many types of defuzzification techniques available in the literature, see [7] for a good overview. In this work, we use the following defuzzifier extensively.

Example 1. For an $A \in \mathcal{F}(X)$, the Mean of Maxima (MeOM) defuzzifier gives as output the mean of all those values in $X$ with the highest membership value, which can be mathematically expressed as

$$
\operatorname{MeOM}(A)=\frac{\sum_{x \in \operatorname{Ceil} A} x}{|\operatorname{Ceil}(A)|} \text { or } \frac{\int_{x \in \operatorname{Ceil} A} d x}{|\operatorname{Ceil}(A)|} .
$$

depending on whether Ceil $A$ is finite or not. 


\section{$2.3 \quad$ Fuzzy Rules}

Given two non-empty classical sets $X, Y \subseteq \mathbb{R}$, a Single-Input Single-Output (SISO) fuzzy IF-THEN rulebase consists of rules of the form:

$$
\text { IF } \tilde{x} \text { is } A_{i} \text { THEN } \tilde{y} \text { is } B_{i},
$$

where $\tilde{x}, \tilde{y}$ are the linguistic variables and $A_{i}, B_{i}, i=1,2, \ldots n$ are the linguistic values taken by the linguistic variables. These linguistic values are represented by fuzzy sets in their corresponding domains, i.e., $A_{i} \in \mathcal{F}(X), B_{i} \in \mathcal{F}(Y)$.

Definition 7 ([8]). A fuzzy rule base (3) is called monotone if for any two rules :

$$
\begin{aligned}
& \text { IF } \tilde{x} \text { is } A_{i} \text { THEN } \tilde{y} \text { is } B_{i} \\
& \boldsymbol{I F} \tilde{x} \text { is } A_{j} \text { THEN } \tilde{y} \text { is } B_{j},
\end{aligned}
$$

such that $A_{i} \prec A_{j}$, the inequality $B_{i} \prec B_{j}$ holds.

\subsection{Fuzzy Logic Connectives}

Definition 8 ( [1], Definition 1.1.1). A function $I:[0,1]^{2} \longrightarrow[0,1]$ is called a fuzzy implication if it is decreasing in the first variable, increasing in the second variable and $I(0,0)=1, I(1,1)=1, I(1,0)=0$.

Yager 9] introduced a family of fuzzy implications based on strictly monotonic functions on $[0,1]$.

Definition 9 ( [1], Definition 3.1.1). Let $f:[0,1] \rightarrow[0, \infty]$ be a strictly decreasing and continuous function with $f(1)=0$. The function $I_{f}:[0,1]^{2} \rightarrow[0,1]$ defined by

$$
I_{f}(x, y)=f^{-1}(x \cdot f(y)), \quad x, y \in[0,1],
$$

with the understanding $0 \cdot \infty=0$, is a fuzzy implication and called an $f$-implication.

The function $f$ itself is called an $f$-generator of the $I_{f}$ generated as in (4). We will often write $\longrightarrow_{f}$ instead of $I_{f}$. We can divide the whole class of $f$-implication into two classes depending on whether $f(0)=\infty$ or $f(0)<\infty$. In this article we only consider the class of $f$-implications for which $f(0)=\infty$, in which case, it is easy to verify that $x \longrightarrow{ }_{f} 0=0$ for any $x \in(0,1]$.

\section{Fuzzy Relational Inference Mechanism}

Given a rule base of the form (3) and an input " $\tilde{x}$ is $A^{\prime}$ ", the main objective of a fuzzy inference mechanism is to find a meaningful $B^{\prime}$ such that " $\tilde{y}$ is $B^{\prime}$ ". While many types of fuzzy inference mechanisms have been proposed in the literature we restrict this study only to Fuzzy Relation based inference mechanisms.

The inference mechanism in a fuzzy relational inference (FRI) can be expressed as follows:

$$
B^{\prime}=f_{R}^{@}\left(A^{\prime}\right)=A^{\prime} @ R,
$$

where $A^{\prime} \in \mathcal{F}(X)$ is the input, the relation $R: X \times Y \rightarrow[0,1]$ i.e, $R \in \mathcal{F}(X \times Y)$ represents or models the rule base, $B^{\prime}$ is the obtained output and @ is called the composition operator, which is a mapping @: $\mathcal{F}(X) \times \mathcal{F}(X \times Y) \rightarrow \mathcal{F}(Y)$. 


\subsection{FRI with Singleton Input : $f_{R}^{@} \equiv f_{R}$}

Often one needs to deal with crisp inputs $x_{0} \in X$. In such a case the given input is suitably fuzzified, i.e., a fuzzy set $A^{\prime} \in \mathcal{F}(X)$ is suitably constructed from $x_{0}$. Commonly, the following singleton fuzzifier is employed:

$$
A^{\prime}(x)= \begin{cases}1, & x=x_{0} \\ 0, & x \neq x_{0}\end{cases}
$$

With the above input $A^{\prime}$, the FRI mechanism (FRI-R reduces to

$$
B^{\prime}(y)=R\left(x_{0}, y\right), \quad y \in Y .
$$

(FRI-R-Singleton)

Thus in the case of a singleton input, the output is fully dependent on the model of the rule base $R$. In other words, $f_{R}^{@} \equiv f_{R}$ and hence the composition @ does not play any role.

We denote an FRI with singleton input as a quadruple $\mathbb{F}=\left(\left\{A_{i}, B_{i}\right\}, R, g\right)$, where $A_{i}$ 's and $B_{i}$ 's correspond to the input and output fuzzy partitions on $X$ and $Y$, respectively, $R$ is the fuzzy relation modeling the rule base and $g$ is the defuzzifier used to obtain a crisp output from the obtained $B^{\prime}$.

\subsection{FRI with Yager's Class of Fuzzy Implications}

In this work, we consider the Relational inference mechanism, where the fuzzy implication is $f$-implication, one of the Yager's classes of fuzzy implications. Essentially, we interpret the $\longrightarrow$ in (FRI- $R$-Singleton as an $f$-implication with $f(0)=\infty$ and consider the following relation $R$ to represent the rule base:

$$
\hat{R}_{f}(x, y)=\bigwedge_{i=1}^{n}\left(A_{i}(x) \longrightarrow_{f} B_{i}(y)\right), \quad x \in X, y \in Y . \quad\left(\operatorname{Imp}-\hat{R}_{f}\right)
$$

Recently, we have studied the suitability of the Bandler-Kohout Subproduct FRI with $\left[\mathrm{Imp}-\hat{R}_{f}\right)$ representing the implicative form of the rule base in [5]. In this work, we consider $\left(\underline{\operatorname{Imp}}-\hat{R}_{f}\right)$ and discuss the issue of monotonicity in this context.

\section{Monotonicity of FRI with $f$-Implication(SISO Case)}

Theorem 1. Let us consider an FRI $\mathbb{F}=\left(\left\{A_{i}, B_{i}\right\}, R, g\right)$ with $R=\hat{R}_{f}$ with a monotone rule base of the form (3) such that $\left\{A_{i}\right\}_{i=1}^{n},\left\{B_{i}\right\}_{i=1}^{n}$ are normal, convex, strict on both sides of the ceiling and form a Ruspini Partition on $X$ and $Y$ respectively. Then $\mathbb{F}$ with MeOM defuzzification as in (2) is monotonic.

Proof. While the proof is valid for any fuzzy sets which are normal, convex and strict on both sides of ceiling, for better readability we prove this result for triangular fuzzy sets only. Fuzzy relational inference mechanism (FRI- $R$-Singleton) with $R=\hat{R}_{f}$ is of the form,

$$
B^{\prime}(y)=\hat{R}_{f}\left(x_{0}, y\right), \quad y \in Y
$$


Let the convex fuzzy sets $\left\{A_{i}\right\}_{i=1}^{n},\left\{B_{i}\right\}_{i=1}^{n}$ be such that Supp $A_{i}=\left[x_{i-1}, x_{i+1}\right]$, Supp $B_{i}=\left[y_{i-1}, y_{i+1}\right]$ and $A_{i}\left(x_{i}\right)=1, B_{i}\left(y_{i}\right)=1$. Let $x^{\prime}, x^{\prime \prime} \in X$ be the two given inputs such that $x^{\prime} \leq x^{\prime \prime}$. We consider the following two cases and show that the obtained outputs $y^{\prime}, y^{\prime \prime} \in Y$ also are similarly ordered, i.e., $y^{\prime} \leq y^{\prime \prime}$.

Case-1: $x^{\prime}, x^{\prime \prime} \in\left[x_{i}, x_{i+1}\right]$, for $i=1,2, \ldots, n-1$.

Since $\left\{A_{i}\right\}_{i=1}^{n}$ form Ruspini partition $A_{j}\left(x^{\prime}\right)=0 \forall j \neq i, i+1$. From (5),

$$
B^{\prime}(y)=\left[A_{i}\left(x^{\prime}\right) \longrightarrow_{f} B_{i}(y)\right] \wedge\left[A_{i+1}\left(x^{\prime}\right) \longrightarrow_{f} B_{i+1}(y)\right] .
$$

Let $A_{i}\left(x^{\prime}\right)=\alpha_{i}^{\prime}$ and $A_{i+1}\left(x^{\prime}\right)=\alpha_{i+1}^{\prime}$. Since $A_{i}$ 's form a Ruspini partition, $\alpha_{i}^{\prime}+\alpha_{i+1}^{\prime}=$ 1. Similarly, with $A_{i}\left(x^{\prime \prime}\right)=\alpha_{i}^{\prime \prime}$ and $A_{i+1}\left(x^{\prime \prime}\right)=\alpha_{i+1}^{\prime \prime}$, we have $\alpha_{i}^{\prime \prime}+\alpha_{i+1}^{\prime \prime}=1$. Thus,

$$
\begin{aligned}
B^{\prime}(y) & =\left[\alpha_{i}^{\prime} \longrightarrow_{f} B_{i}(y)\right] \wedge\left[\alpha_{i+1}^{\prime} \longrightarrow_{f} B_{i+1}(y)\right]=B_{i}^{\prime}(y) \wedge B_{i+1}^{\prime}(y), \\
B^{\prime \prime}(y) & =\left[\alpha_{i}^{\prime \prime} \longrightarrow_{f} B_{i}(y)\right] \wedge\left[\alpha_{i+1}^{\prime \prime} \longrightarrow_{f} B_{i+1}(y)\right]=B_{i}^{\prime \prime}(y) \wedge B_{i+1}^{\prime \prime}(y)
\end{aligned}
$$

Now since $A_{i}$ is decreasing in $\left[x_{i}, x_{i+1}\right]$ and $A_{i+1}$ is increasing in $\left[x_{i}, x_{i+1}\right]$,

$$
x^{\prime} \leq x^{\prime \prime} \text { and } x^{\prime}, x^{\prime \prime} \in\left[x_{i}, x_{i+1}\right] \Longrightarrow A_{i}\left(x^{\prime}\right) \geq A_{i}\left(x^{\prime \prime}\right) \text { and } A_{i+1}\left(x^{\prime}\right) \leq A_{i+1}\left(x^{\prime \prime}\right) .
$$

i.e., $\alpha_{i}^{\prime} \geq \alpha_{i}^{\prime \prime}$ and $\alpha_{i+1}^{\prime} \leq \alpha_{i+1}^{\prime \prime}$. Clearly, since $B_{i}, B_{i+1}$ are convex and normal, $B_{i}^{\prime}, B_{i+1}^{\prime}$ are also convex and normal. So $B^{\prime}=B_{i}^{\prime} \cap B_{i+1}^{\prime}$ and $B^{\prime \prime}=B_{i}^{\prime \prime} \cap B_{i+1}^{\prime \prime}$ are also convex. Let $y^{\prime}=\operatorname{MeOM}\left(B^{\prime}\right)$ and $y^{\prime \prime}=\operatorname{MeOM}\left(B^{\prime \prime}\right)$.

Claim: If $x^{\prime} \in\left[x_{i}, x_{i+1}\right]$, then $y^{\prime} \in\left[y_{i}, y_{i+1}\right]$ for $i=1,2, \ldots, n-1$.

Now since $x \longrightarrow_{f} 0=0$ for any $x \in(0,1]$, we have that the supports of both the modified fuzzy sets $B_{i}^{\prime}=\alpha_{i} \longrightarrow_{f} B_{i}, B_{i+1}^{\prime}=\alpha_{i+1} \longrightarrow_{f} B_{i+1}$ are the same as those of $B_{i}, B_{i+1}$, i.e., Supp $B_{i}^{\prime}=\operatorname{Supp} B_{i}$ and Supp $B_{i+1}^{\prime}=\operatorname{Supp} B_{i+1}$. Hence,

$$
\begin{aligned}
\operatorname{Supp} B^{\prime} & =\operatorname{Supp} B_{i}^{\prime} \cap \operatorname{Supp} B_{i+1}^{\prime}=\operatorname{Supp} B_{i} \cap \operatorname{Supp} B_{i+1} \\
& =\operatorname{Supp}\left(B_{i} \cap B_{i+1}\right)=\left[y_{i}, y_{i+1}\right] .
\end{aligned}
$$

Since (6) holds we have,

$$
y^{\prime}=g\left(x^{\prime}\right)=\operatorname{MeOM}\left(B^{\prime}(y)\right) \in \operatorname{Supp}\left(B_{i} \cap B_{i+1}\right)=\left[y_{i}, y_{i+1}\right] .
$$

So, $y^{\prime}, y^{\prime \prime} \in\left[y_{i}, y_{i+1}\right]$ and we obtain the following inequalities, for any $y \in\left[y_{i}, y_{i+1}\right]$ :

$$
\begin{aligned}
\alpha_{i}^{\prime} \geq \alpha_{i}^{\prime \prime} & \Longrightarrow \alpha_{i}^{\prime} \longrightarrow_{f} B_{i}(y) \leq \alpha_{i}^{\prime \prime} \longrightarrow_{f} B_{i}(y), \\
& \Longrightarrow B_{i}^{\prime}(y) \leq B_{i}^{\prime \prime}(y) .
\end{aligned}
$$

Similarly, $\alpha_{i+1}^{\prime} \leq \alpha_{i+1}^{\prime \prime} \Longrightarrow \alpha_{i+1}^{\prime} \longrightarrow_{f} B_{i+1}(y) \geq \alpha_{i+1}^{\prime \prime} \longrightarrow_{f} B_{i+1}(y)$,

$$
\Longrightarrow B_{i+1}^{\prime}(y) \geq B_{i+1}^{\prime \prime}(y) \text {. }
$$

Since $B_{i}$ 's are triangular fuzzy sets, $y^{\prime}, y^{\prime \prime} \in\left[y_{i}, y_{i+1}\right]$ are the points which satisfy

$$
\begin{aligned}
& B_{i}^{\prime}\left(y^{\prime}\right)=B_{i+1}^{\prime}\left(y^{\prime}\right) \Longrightarrow \alpha_{i}^{\prime} \longrightarrow_{f} B_{i}\left(y^{\prime}\right)=\alpha_{i+1}^{\prime} \longrightarrow_{f} B_{i+1}\left(y^{\prime}\right), \\
& B_{i}^{\prime \prime}\left(y^{\prime \prime}\right)=B_{i+1}^{\prime \prime}\left(y^{\prime \prime}\right) \Longrightarrow \alpha_{i}^{\prime \prime} \longrightarrow B_{i}\left(y^{\prime \prime}\right)=\alpha_{i+1}^{\prime \prime} \longrightarrow_{f} B_{i+1}\left(y^{\prime \prime}\right) .
\end{aligned}
$$

Now, to prove monotonicity, we need to show that $y^{\prime} \leq y^{\prime \prime}$.

If possible, let us assume to the contrary that $y^{\prime}>y^{\prime \prime}$. Since $B_{i}$ and $B_{i+1}$ are, respectively, strictly decreasing and strictly increasing on $\left[y_{i}, y_{i+1}\right]$,

$$
y^{\prime}>y^{\prime \prime} \text { and } y^{\prime}, y^{\prime \prime} \in\left[y_{i}, y_{i+1}\right] \Longrightarrow B_{i}\left(y^{\prime}\right)<B_{i}\left(y^{\prime \prime}\right) \text { and } B_{i+1}\left(y^{\prime}\right)>B_{i+1}\left(y^{\prime \prime}\right) \text {. }
$$


This leads to the following inequalities:

$$
\begin{array}{rlrl}
\alpha_{i+1}^{\prime} \longrightarrow_{f} B_{i+1}\left(y^{\prime}\right) & >\alpha_{i+1}^{\prime} \longrightarrow_{f} B_{i+1}\left(y^{\prime \prime}\right) & \left(\text { Since } \longrightarrow_{f}\right. & \text { is strict and using (9)) } \\
& \geq \alpha_{i+1}^{\prime \prime} \longrightarrow_{f} B_{i+1}\left(y^{\prime \prime}\right) & \left(\text { Since } \alpha_{i+1}^{\prime} \leq \alpha_{i+1}^{\prime \prime}\right) \\
& =\alpha_{i}^{\prime \prime} \longrightarrow_{f} B_{i}\left(y^{\prime \prime}\right) & \text { (Using (8) ) } \\
& >\alpha_{i}^{\prime \prime} \longrightarrow_{f} B_{i}\left(y^{\prime}\right) & \text { (Using (9)) } \\
& \geq \alpha_{i}^{\prime} \longrightarrow_{f} B_{i}\left(y^{\prime}\right), & \left(\text { Since } \alpha_{i}^{\prime} \geq \alpha_{i}^{\prime \prime}\right)
\end{array}
$$

i.e., $\alpha_{i+1}^{\prime} \longrightarrow_{f} B_{i+1}\left(y^{\prime}\right)>\alpha_{i}^{\prime} \longrightarrow_{f} B_{i}\left(y^{\prime}\right)$, a contradiction to (7). So we have $x^{\prime} \leq$ $x^{\prime \prime} \Longrightarrow y^{\prime} \leq y^{\prime \prime}$.

Case-2: Let $x^{\prime} \in\left[x_{i}, x_{i+1}\right]$ and $x^{\prime \prime} \in\left[x_{i+p}, x_{i+p+1}\right], p \geq 1$. By the claim above, we have $y^{\prime} \in\left[y_{i}, y_{i+1}\right]$ and $y^{\prime \prime} \in\left[y_{i+p}, y_{i+p+1}\right], p \geq 1$ and hence $y^{\prime} \leq y^{\prime \prime}$.

\section{Concluding Remarks}

FRIs are one of the most important inference mechanisms to be studied. However, all of the known works on FRIs employ operations that come from a residuated lattice structure. In this work, we have shown that one of the most desirable properties, viz., monotonicity, is available to us with the operations coming from a non-residuated structure.

\section{References}

1. Baczyński, M., Jayaram, B.: Fuzzy Implications. STUDFUZZ, vol. 231, pp. 1-35. Springer, Heidelberg (2008)

2. Bandler, W., Kohout, L.J.: Semantics of implication operators and fuzzy relational products. Internat. J. Man-Mach. Stud. 12, 89-116 (1980)

3. Broekhoven, E.V., Baets, B.D.: Only Smooth Rule Bases Can Generate Monotone Mamdani-Assilian Models Under Center-of-Gravity Defuzzification. IEEE Transactions on Fuzzy Systems 17, 1157-1174 (2009)

4. Mamdani, E.H., Assilian, S.: An experiment in linguistic synthesis with a fuzzy logic contoller. Int. J. Man-Machine Studies 7, 1-13 (1975)

5. Mandal, S., Jayaram, B.: Bandler-Kohout Subproduct with Yagers classes of Fuzzy Implications. IEEE Trans. on Fuzzy Syst. (accepted 2013)

6. Pedrycz, W.: Application of fuzzy relational equations for methods of reasoning in presence of fuzzy data. Fuzzy Sets and Syst. 16, 163-175 (1985)

7. Roychowdhury, S., Pedrycz, W.: A survey of defuzzification strategies. International Journal of Intelligent Systems 16, 679-695 (2001)

8. Štěpnička, M., De Baets, B.: Monotonicity of implicative fuzzy models. In: IEEE International Conference on Fuzzy Systems, pp. 1-7 (2010)

9. Yager, R.R.: On some new classes of implication operators and their role in approximate reasoning. Inform. Sci. 167, 193-216 (2004)

10. Zadeh, L.A.: Outline of a new approach to the analysis of complex systems and decision processes. IEEE Trans. on Syst. Man and Cyber. SMC-3(1), 28-44 (1973) 